\title{
A well-differentiated liposarcoma of the prevertebral space: a case report
}

\author{
Tianming Yang^, Lin Xiao^, Huijun Ren^ \\ Department of Otolaryngology, Taizhou Hospital of Zhejiang Province Affiliated to Wenzhou Medical University, Linhai, China \\ Correspondence to: Lin Xiao. Bachelor's degree, Department of Otolaryngology, Taizhou Hospital of Zhejiang Province Affiliated to Wenzhou Medical \\ University, Ximen Street, Linhai, China. Email: 798768696@qq.com.
}

\begin{abstract}
Liposarcoma is common in adults; however, it very rarely occurs in the retropharyngeal space, and to date, no cases of liposarcoma in the prevertebral space have been reported. A 78-year-old man presented at the Otolaryngology Department with a 1-month history of dyspnea and dysphonia. Magnetic resonance imaging of the neck reveled a retropharyngeal mass extending from the superior margin of the axis to the level of the $7^{\text {th }}$ cervical vertebra. A computed tomography scan of the patient's neck uncovered the relationship between the mass and its' surrounding structures. Based on the above examinations, a diagnosis of retropharyngeal liposarcoma was made initially. The patient underwent transoral surgical excision with the assistance of a nasal endoscope. During surgery, the tumor was located in the prevertebral space. Combined with pathology and immunohistochemistry examinations, the diagnosis was modified to well-differentiated liposarcoma of the prevertebral space. At the 1-year follow-up, the patient was well and there was no evidence of recurrence. The long-term outcomes are not yet known. Liposarcoma of the prevertebral space may sometimes be misdiagnosed as retropharyngeal liposarcoma; however, in our view, it is not necessary to focus on differentiating between enormous retropharyngeal and prevertebral tumors. In relation to the short-term outcome, complete excision using the transoral approach with the aid of nasal endoscope may present a better choice than the cervical approach in treating these tumors, especially in old people.
\end{abstract}

Keywords: Liposarcoma; prevertebral space; retropharyngeal space; transoral approach; case report

Submitted Jan 23, 2021. Accepted for publication Mar 06, 2021.

doi: $10.21037 /$ tcr-21-143

View this article at: http://dx.doi.org/10.21037/tcr-21-143

\section{Introduction}

Liposarcoma is among the most common types of soft tissue sarcoma in adults, and usually occurs in the lower extremities, retroperitoneum, perinephric space, mesentery area, or shoulders. The incidence of liposarcoma in the head and neck region, at approximately $1.8-6.2 \%$, is extremely low (1). A search of the PubMed database indicated that to date, no cases of liposarcoma in the prevertebral space have been reported.

Retropharyngeal liposarcoma can produce lifeendangering pressure symptoms, such as dysphagia, dyspnea and dysphonia. Due to the complex anatomic situation, radical resection represents a significant challenge in patients with retropharyngeal liposarcoma. Further, the prognosis of this disease is poor. In the case described herein, due to concerns that the patient was too old to tolerate the operation, transoral surgical excision with the assistance of a nasal endoscope was proposed. This approach may provide a novel solution for surgeons seeking to treat retropharyngeal and prevertebral tumors.

In this paper, we report the first case of a giant liposarcoma located in the prevertebral space. We hope that our experience will increase surgeons' awareness

^ ORCID: Tianming Yang, 0000-0002-0331-0712; Lin Xiao, 0000-0002-3688-3939; Huijun Ren, 0000-0001-7257-7501. 

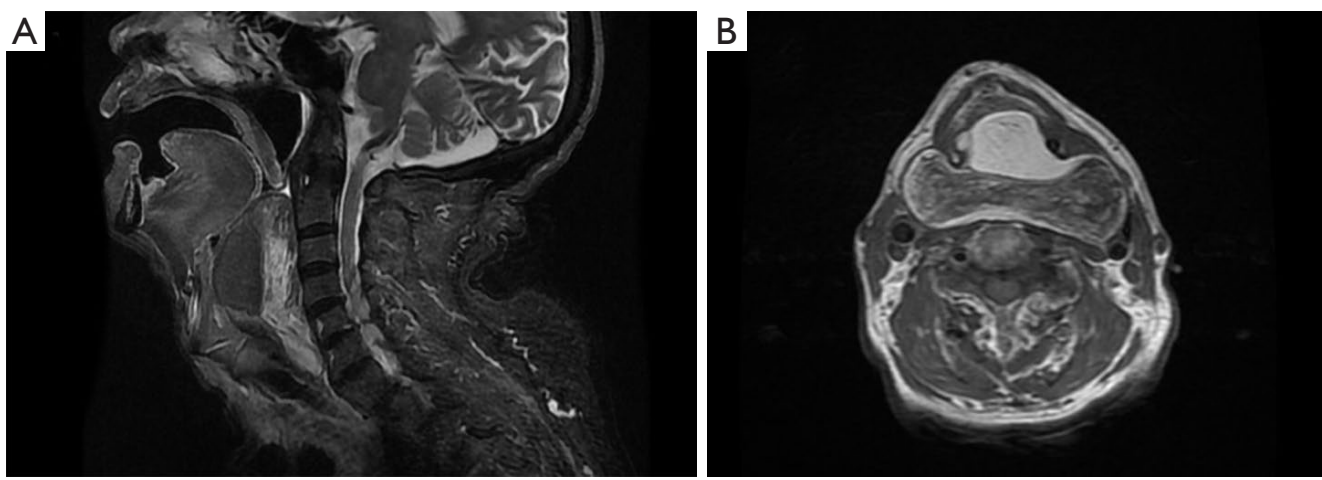

Figure 1 Magnetic resonance imaging (MRI) scans of the patient. (A) MRI scan of the patient's neck showing a retropharyngeal mass with a fatty/solid consistency, extending from the superior margin of the axis to the level of the $7^{\text {th }}$ cervical vertebra, measuring $9.4 \mathrm{~cm} \times 7.9 \mathrm{~cm}$ $\times 3.9 \mathrm{~cm}$, and narrowing the laryngopharynx. (B) MRI scan of the patient's neck showing a retropharyngeal mass exhibiting no obvious enhancement with a contrast agent.

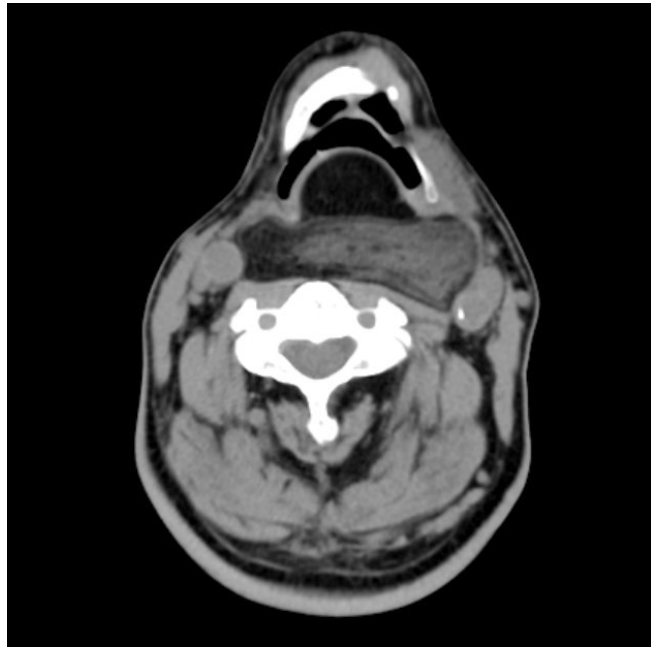

Figure 2 Computed tomography (CT) scans of the patient's neck showing the relationship between the epiglottis and the mass.

of liposarcoma of the prevertebral space and provide diversified methods for treating retropharyngeal and prevertebral tumors.

We present the following article in accordance with the CARE reporting checklist (available at http://dx.doi. org/10.21037/tcr-21-143).

\section{Case presentation}

A 78-year-old man presented to the Otolaryngology Department after experiencing dyspnea and dysphonia for 1 month. He also had a 1-year history of progressive dysphagia. His remaining medical, surgical, family, and social history were noncontributory except that he had undergone a thyroidectomy treat a nodular goiter 6 years previously. A physical examination of the patient found no cervical swelling or palpable masses. An oropharyngeal examination showed a bulge in the posterior pharyngeal wall. As the mass was extremely large, electronic laryngoscopy failed, and it only showed a massive bulge in the posterior pharyngeal wall occupying almost the entirety of the pharyngeal space. Magnetic resonance imaging (MRI) of the patient's neck showed a retropharyngeal mass with a fatty/solid consistency, extending from the superior margin of the axis to the level of the $7^{\text {th }}$ cervical vertebra, measuring $9.4 \mathrm{~cm} \times 7.9 \mathrm{~cm} \times 3.9 \mathrm{~cm}$, and narrowing the laryngopharynx (Figure 1A). Contrast-enhanced MRI of the neck was performed to further characterize the mass and revealed a retropharyngeal mass that exhibited no obvious enhancement with a contrast agent (Figure 1B). To further characterize the relationship between the mass and its' surrounding structures, a computed tomography (CT) scan was performed. The scan showed no infringement of the mass upon any surrounding structures (Figure 2). Based on the above examinations, a clinical diagnosis of retropharyngeal liposarcoma was made initially.

Complete excision of the mass represented a challenge for the surgeon. Due to the patient's age, it was thought that a trans-cervical approach may cause trauma. Further, the mass extended up to the $7^{\text {th }}$ cervical vertebra. After some discussion, a transoral surgical excision was planned with the assistance of a nasal endoscope. As tracheal intubation would have been impossible even with a fiberoptic 

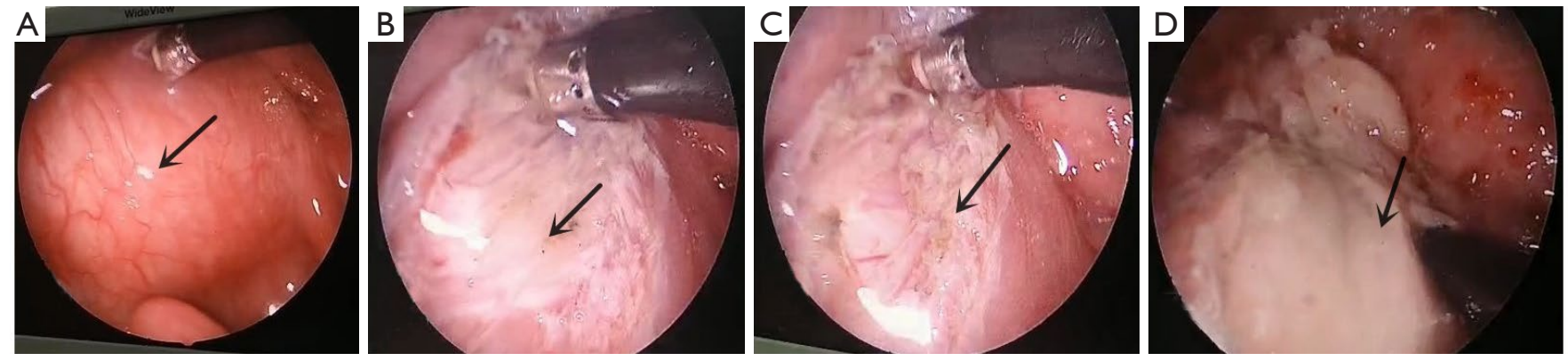

Figure 3 Pictures of surgical procedures. The arrows show (A) the mass in the posterior pharyngeal wall, and (B) the constrictor of pharynx. The constrictor of pharynx (C) and the prevertebral fascia (D) were cut off.
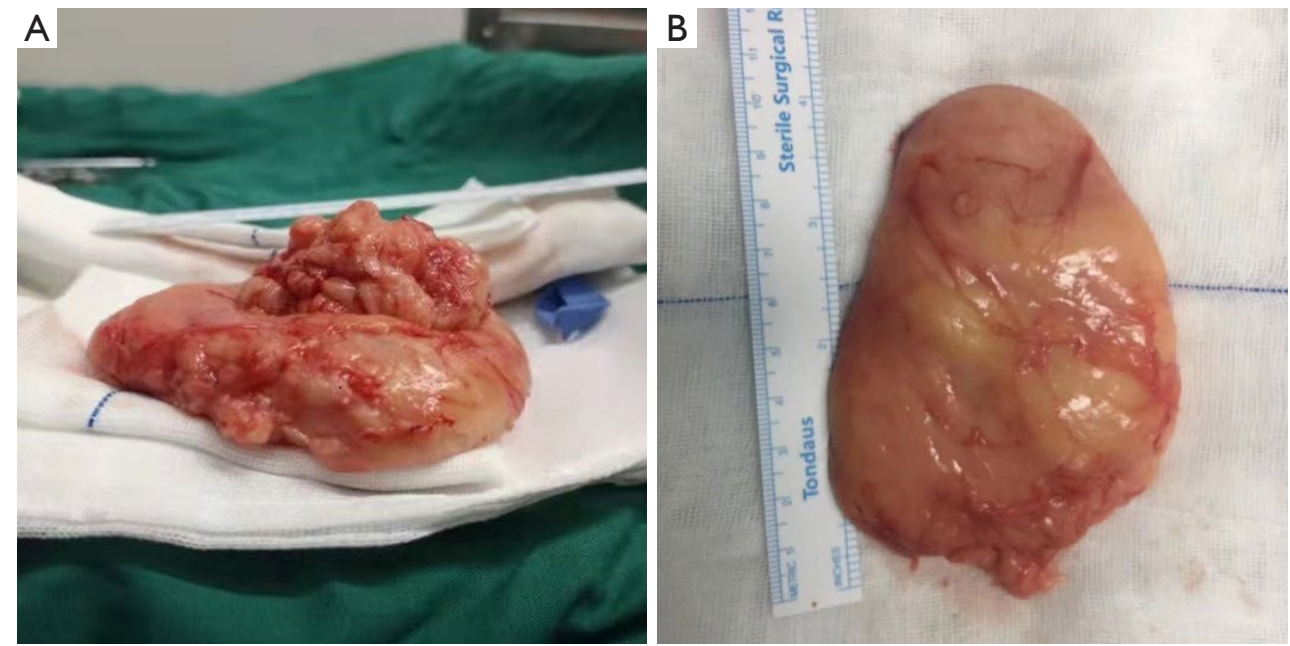

Figure 4 The excised tumor. (A) The excised tumor and a pile of broken tissue measuring $3.5 \mathrm{~cm} \times 3.2 \mathrm{~cm} \times 1.2 \mathrm{~cm}$. (B) The excised tumor was yellow, defined, and soft, measured $9.0 \mathrm{~cm} \times 8.5 \mathrm{~cm} \times 1.5 \mathrm{~cm}$ in size, and had a fibrous capsule.

bronchoscope, the tracheostomy was performed under local anesthesia. The operation was then performed under general anesthesia. First, a longitudinal incision was made at the surface of the mass (Figure $3 A$ ). Next, the mucous membrane, constrictor of the pharynx (Figure $3 B$ ), and buccopharyngeal fascia were cut off (Figure 3C), but the tumor could not be seen. After cutting off the prevertebral fascia (Figure 3D), the tumor was found between the prevertebral fascia and the cervical spine. Finally, the tumor was successfully removed with no damage sustained to the surrounding tissue, and the incision was sutured layer by layer. The tumor was yellow, defined, and soft, and measured $9.0 \mathrm{~cm} \times 8.5 \mathrm{~cm} \times 1.5 \mathrm{~cm}$ in size with a fibrous capsule (Figure 4). Pathology and immunohistochemistry revealed the tumor to be an atypical lipomatous tumor/well- differentiated liposarcoma. Consequently, the diagnosis was modified to well-differentiated liposarcoma of the prevertebral space. One week later, the tracheostomy tube was plugged, and the patient experienced no more dyspnea or dysphonia. The tracheostoma was sutured 72 hours after that. Two weeks later, the patient was allowed to consume semi-fluid foods and did well swallowing. At the 3-month postoperative follow-up, we performed a CT scan of the neck to evaluate the patient's condition, and found no signs of recurrence (Figure 5). At the 1-year postoperative followup, the patient continued to do well, and there was no evidence of recurrence. The patient's short-term prognosis is excellent, but the long-term outcomes are not yet known.

All procedures performed in studies involving human participants were in accordance with the ethical standards of 


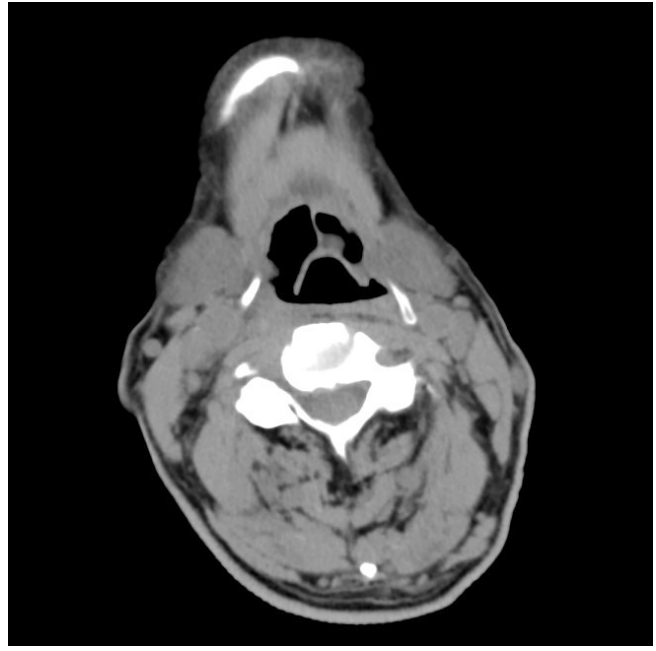

Figure 5 CT scan of the patient's neck showing no signs of recurrence at 3 months after surgery.

the institutional and/or national research committee(s) and with the Helsinki Declaration (as revised in 2013). Written informed consent was obtained from the patient.

\section{Discussion}

There are 3 fascia layers in the retropharyngeal area: the oropharyngeal layer, the buccopharyngeal fascia, and the prevertebral fascia. The retropharyngeal space lies between the buccopharyngeal fascia and the prevertebral fascia, and extends from the skull base to the mediastinum. The prevertebral space is located between the prevertebral fascia and the vertebra, and extends from the skull base to the $3^{\text {rd }}$ thoracic vertebra. Patients with liposarcoma are usually asymptomatic unless the tumor grows to an enormous size causing cosmetic deformity or pressure effects. Also, symptoms vary depending on the anatomical location and size of the tumor. Retroperitoneal liposarcomas are usually asymptomatic due to the anatomic location. When a retroperitoneal liposarcoma grows to an extremely large size, it can present as an asymptomatic abdominal mass or cause pressure symptoms such as abdominal pain/fullness, flank pain or early satiety. Complete surgical resection with negative microscopic margins is the main treatment for retroperitoneal liposarcoma. Even with grossly complete resection, the disease has a high recurrence rate and a poor prognosis. Thus, long-term follow-up appointments are required (2).

Liposarcomas in the retropharyngeal space can produce pressure symptoms such as dysphagia, dyspnea and dysphonia. As the retropharyngeal and prevertebral spaces are adjacent, prevertebral tumors can cause similar symptoms. As the fascia layers cannot be directly seen in the images, and the anatomic locations of the retropharyngeal space and prevertebral space are adjacent, it is difficult to distinguish between retropharyngeal and prevertebral tumors using imaging. The primary tumor site can be estimated based on the surrounding structures of the tumor, such as the posterior pharyngeal space fat, the prevertebral muscle and the carotid sheath. For instance, retropharyngeal tumors often cause prevertebral muscles to move inward, while prevertebral tumors often cause prevertebral muscles to move forward. Further, retropharyngeal tumors are often confined to the left or right side. As the retropharyngeal space is divided into left and right sides by the pharyngeal raphe at the midline, the 2 sides are not connected to each other. However, if the retropharyngeal tumor is enormous, it may not be confined to 1 side, and the features of the surrounding structures may not be clear. In this situation, liposarcoma of the prevertebral space may sometimes be misdiagnosed as retropharyngeal liposarcoma. Therefore, surgery may be the only way to distinguish between enormous retropharyngeal and prevertebral tumors. Thus, in our view, it is not necessary to focus on differentiating between enormous retropharyngeal and prevertebral tumors.

Although a preliminary diagnosis of liposarcoma can be made by CT or MRI, pathology and immunohistochemistry examinations are still needed for diagnostic confirmation. Due to the small number of cases of liposarcoma of the head and neck, the management of these tumors has largely been based on experience with limb and retroperitoneal tumors. The main treatment for liposarcoma is surgical excision with adequate margins (2). However, given the abundance of complex and vital neurovascular and functional upperaero digestive structures, attaining adequate margins in the head and neck region is difficult. Further, for a liposarcoma in lymph nodes with a relatively low metastasis rate, routine neck dissection is not recommended. It is our view that transoral surgical excision with the assistance of a nasal endoscope may be more beneficial than the cervical approach. The indication for adjuvant radiation therapy is still unclear, some authors (3) believe that wide surgical excision alone is sufficient for successful treatment. However, others (4-6) have recommended adjuvant radiotherapy, as they believe that liposarcomas often infiltrate the surrounding structures microscopically, even 
if they have a clear fibrous capsule. In the present case, the patient was advised that the tumor may recur, but he refused to undergo adjuvant radiotherapy, as he could not afford follow-up treatment. At 3 months after the surgery, we performed a CT scan of the neck to evaluate the patient's condition and found no signs of recurrence (Figure 5). At 1-year after the initial surgical procedure, the patient continued to do well, and there was no evidence of recurrence. Thus, in terms of the short-term outcome, we believe complete excision using the transoral approach may be a good choice to treat liposarcoma in the prevertebral space, especially in patients of advanced age.

\section{Acknowledgments}

Funding: None.

\section{Footnote}

Reporting Checklist: The authors have completed the CARE reporting checklist. Available at http://dx.doi.org/10.21037/ tcr-21-143

Conflicts of Interest: All authors have completed the ICMJE uniform disclosure form (available at http://dx.doi. org/10.21037/tcr-21-143). The authors have no conflicts of interest to declare.

Ethical Statement: The authors are accountable for all aspects of the work in ensuring that questions related to the accuracy or integrity of any part of the work are appropriately investigated and resolved. All procedures performed in studies involving human participants were in accordance with the ethical standards of the institutional and/or national research committee(s) and with the Helsinki Declaration (as revised in 2013). Written informed consent was obtained from the patient.

Open Access Statement: This is an Open Access article distributed in accordance with the Creative Commons Attribution-NonCommercial-NoDerivs 4.0 International License (CC BY-NC-ND 4.0), which permits the noncommercial replication and distribution of the article with the strict proviso that no changes or edits are made and the original work is properly cited (including links to both the formal publication through the relevant DOI and the license). See: https://creativecommons.org/licenses/by-nc-nd/4.0/.

\section{References}

1. Yueh B, Bassewitz HL, Eisele DW. Retropharyngeal liposarcoma. Am J Otolaryngol 1995;16:331-40.

2. Vijay A, Ram L. Retroperitoneal liposarcoma: a comprehensive review. Am J Clin Oncol 2015;38:213-9.

3. Tan MC, Brennan MF, Kuk D, et al. Histology-based Classification Predicts Pattern of Recurrence and Improves Risk Stratification in Primary Retroperitoneal Sarcoma. Ann Surg 2016;263:593-600.

4. Ozawa H, Soma K, Ito M, et al. Liposarcoma of the retropharyngeal space: report of a case and review of literature. Auris Nasus Larynx 2007;34:417-21.

5. Takano K, Kondoh A, Matsumiya H, et al. A welldifferentiated liposarcoma of the hypopharynx. Otolaryngol Head Neck Surg 2011;144:479-80.

6. Gleinser DM, Font JP, Clement CG, et al. Primary myxoid liposarcoma of the supraglottic larynx. Rare Tumors 2010;2:e41.
Cite this article as: Yang T, Xiao L, Ren H. A welldifferentiated liposarcoma of the prevertebral space: a case report. Transl Cancer Res 2021;10(7):3600-3604. doi: 10.21037/ tcr-21-143 